\title{
Pediatric Antibiotic-refractory Lyme Arthritis: A Multicenter Case-control Study
}

\author{
Daniel B. Horton (iD, Alysha J. Taxter, Amy L. Davidow (iD, Brandt Groh, David D. Sherry, \\ and Carlos D. Rose
}

\begin{abstract}
Objective. Few factors have consistently been linked to antibiotic-refractory Lyme arthritis (ARLA). We sought to identify clinical and treatment factors associated with pediatric ARLA.

Methods. We performed a case-control study in 3 pediatric rheumatology clinics in a Lyme-endemic region (2000-2013). Eligible children were aged $\leq 18$ years with arthritis and had positive testing for Lyme disease by Western blot. Cases were 49 children with persistently active arthritis despite $\geq 8$ weeks of oral antibiotics or $\geq 2$ weeks of parenteral antibiotics; controls were 188 children whose arthritis resolved within 3 months of starting antibiotics. We compared preselected demographic, clinical, and treatment factors between groups using logistic regression.

Results. Characteristics positively associated with ARLA were age $\geq 10$ years, prolonged arthritis at diagnosis, knee-only arthritis, and worsening after starting antibiotics. In contrast, children with fever, severe pain, or other signs of systemic inflammation were more likely to respond quickly to treatment. Secondarily, low-dose amoxicillin and treatment nonadherence were also linked to higher risk of ARLA. Greater antibiotic use for children with ARLA was accompanied by higher rates of treatment-associated adverse events (37\% vs 15\%) and resultant hospitalization (6\% vs $1 \%)$.

Conclusion. Older children and those with prolonged arthritis, arthritis limited to the knees, or poor initial response to antibiotics are more likely to have antibiotic-refractory disease and treatment-associated toxicity. Children with severe symptoms of systemic inflammation have more favorable outcomes. For children with persistently active Lyme arthritis after 2 antibiotic courses, pediatricians should consider starting antiinflammatory treatment and referring to a pediatric rheumatologist. (First Release March 1 2019; J Rheumatol 2019;46:943-51; doi:10.3899/jrheum.180775)
\end{abstract}

Key Indexing Terms:

LYME ARTHRITIS

PEDIATRIC ARTHRITIS

RISK FACTORS

EPIDEMIOLOGIC STUDIES

From the Department of Pediatrics, Rutgers Robert Wood Johnson Medical School, New Brunswick, New Jersey; Rutgers Center for Pharmacoepidemiology and Treatment Science, Institute for Health, Health Care Policy and Aging Research, New Brunswick, New Jersey; Department of Biostatistics - Epidemiology, Rutgers School of Public Health, Piscataway, New Jersey; Department of Pediatrics, Brenner Children's Hospital, Wake Forest Baptist Medical Center, Winston-Salem, North Carolina; Department of Pediatrics, Penn State Milton S. Hershey Medical Center, Hershey, Pennsylvania; Department of Pediatrics, Children's Hospital of Philadelphia, Perelman School of Medicine, University of Pennsylvania, Philadelphia, Pennsylvania; Department of Pediatrics, Nemours/A.I. duPont Hospital for Children, Thomas Jefferson University, Wilmington, Delaware.

This study was funded by the US National Institute of Arthritis and Musculoskeletal and Skin Diseases (NIAMS) of the National Institutes of Health (NIH) under award numbers F32-AR066461, L40-AR070497, and K23-AR070286, and the Eunice Kennedy Shriver National Institute of Child Health and Human Development of the NIH under award number T32-HD064567. The content is solely the responsibility of the authors and does not necessarily represent the official views of the NIH, NIAMS, or the Eunice Kennedy Shriver National Institute of Child Health and Human Development.

Dr. Rose has received grant funding from GSK through a subcontract from the University of Leuven, Leuven, Belgium, for research unrelated to the present study.

D.B. Horton, MD, MSCE, Division of Pediatric Rheumatology, Rutgers Robert Wood Johnson Medical School, and Rutgers Center for
Pharmacoepidemiology and Treatment Science, Institute for Health, Health Care Policy and Aging Research, and Rutgers School of Public Health; A.J. Taxter, MD, MSCE, Brenner Children's Hospital, Wake Forest Baptist Medical Center; A.L. Davidow, PhD, Rutgers School of Public Health; B. Groh, MD, Penn State Milton S. Hershey Medical Center; D.D. Sherry, MD, Children's Hospital of Philadelphia, Division of Pediatric Rheumatology, Perelman School of Medicine, University of Pennsylvania; C.D. Rose, MD, Division of Rheumatology, Nemours/A.I. duPont Hospital for Children, Thomas Jefferson University.

Address correspondence to Dr. D.B. Horton, 112 Paterson St., New Brunswick, New Jersey 08901, USA. E-mail: daniel.horton@rutgers.edu Accepted for publication October 9, 2018.

Lyme disease (LD) is the most common vector-borne disease in the United States and Europe, and its incidence is rising ${ }^{1}$. After erythema migrans, arthritis is the second most common LD-specific manifestation and the predominant feature of late-disseminated disease ${ }^{1,2}$. The Infectious Disease Society of America (IDSA) and the American Academy of Pediatrics recommend treatment of Lyme arthritis (LA) with up to 8 weeks of oral antibiotics or 2-4 weeks of intravenous (IV) antibiotics $^{3,4}$. Most people with LA experience resolution after antibiotics, but about $10 \%$ continue to have arthritis

Personal non-commercial use only. The Journal of Rheumatology Copyright $\odot$ 2019. All rights reserved. 
despite adequate antibiotic treatment - a condition known as antibiotic-refractory LA (ARLA) $)^{5}$. The underlying pathophysiology of ARLA remains unclear, but this condition likely results from a combination of microbial and host-specific factors 5 .

Many studies have investigated factors associated with ARLA, yielding few consistent findings. In a predominantly adult cohort with LA, age and other demographics, symptoms, joint counts, and disease duration were not associated with antibiotic response ${ }^{5}$. Pediatric studies have linked variable findings to ARLA, including female sex ${ }^{6}$, higher Lyme Western blot band count ${ }^{6}$, high platelet count ${ }^{7}$, development of arthritis in additional joints after starting antibiotics ${ }^{8}$, and the presence of inflammatory back pain or enthesitis ${ }^{8}$. However, these findings have not been replicated. Several pediatric studies have suggested that older children might be more likely to have ARLA ${ }^{6,7,8}$; however, this finding did not reach statistical significance in some studies 7,8 and was not identified in others ${ }^{9,10}$. In both children and adults, one feature implicated in ARLA is intraarticular glucocorticoid (IAGC) injection before antibiotic initiation, possibly by impairing early host immune defense $\mathrm{e}^{5,11}$.

Refractory post-LD symptoms sometimes lead to prolonged antibiotic treatment, an approach that has not been proven efficacious for cognitive symptoms ${ }^{12,13}$ or LA ${ }^{14}$. This lack of efficacy along with the risks of prolonged antibiotics are strong incentives to limit the duration of antibiotic treatment for ARLA. Further, several antiinflammatory and antirheumatic agents prescribed by rheumatologists may be used to treat ARLA ${ }^{5,6,7,10}$. To better understand the pathophysiology of ARLA, promote judicious antibiotic usage for LA, and ensure timely referral to specialists, we sought to identify characteristics of individuals least and most likely to respond to guideline-compatible treatment. Given the lack of replicability of prior exploratory research and the risks of false-positive findings in such studies, we identified 13 potential explanatory demographic and clinical variables suspected a priori to be related to the development of ARLA in children (Supplementary Table 1, available with the online version of this article).

\section{MATERIALS AND METHODS}

Study design and setting. We performed a case-control study of children with LA from 3 pediatric rheumatology referral centers [Children's Hospital of Philadelphia (CHOP), Nemours/A.I. duPont Hospital for Children, and Penn State Hershey Children's Hospital] that routinely see children from 4 Lyme-endemic states (Pennsylvania, New Jersey, Delaware, and Maryland).

This study was approved by the institutional review boards of the participating centers (CHOP IRB 14-010818, Nemours \#598679, Hershey STUDY00000431) and Rutgers University (PRO Pro20170002088), with a waiver of consent/assent for this minimal-risk retrospective research.

Study population. The study consisted of children aged $\leq 18$ years at LA diagnosis who were seen in a participating rheumatology clinic between January 1, 2000, and December 31, 2013. Potential participants were screened through queries of electronic health records (EHR) for children evaluated in a pediatric rheumatology clinic with a diagnosis of LD (International Classification of Diseases-9-Clinical Modification code
088.81). Clinical LA diagnosis was confirmed by record review according to these criteria: (1) documented positive serologic Lyme testing with $\geq 5$ IgG bands present on Western blot testing performed using standard methods; and (2) documented arthritis on physical examination or arthrocentesis, with other causes of arthritis excluded. Arthritis on examination was defined by the presence of joint effusion or 2 other signs of inflammation (warmth, tenderness, restricted or painful range of motion) documented by a physician. Although several local clinical laboratories did not routinely perform screening ELISA before Western blot testing as recommended by guidelines ${ }^{3,4}$, children with arthritis and positive Western blot testing alone were routinely treated for LA at participating centers. Therefore, children lacking Lyme ELISA results were included in the study and excluded from sensitivity analyses (see Statistical Analysis below).

Case definition. Cases had ARLA as defined by IDSA guidelines: persistently active LA documented $\geq 2$ months after completion of $\geq 8$ weeks of oral antibiotics (amoxicillin, doxycycline, cefuroxime) or $\geq 2$ weeks of IV antibiotics (ceftriaxone, cefotaxime), with negative synovial fluid Lyme PCR testing if performed (primary case definition) ${ }^{5}$. Because some individuals had prolonged arthritis but did not receive sufficient treatment per IDSA guidelines, sensitivity analyses considered a second set of cases with persistently active arthritis for $\geq 6$ months after initiating antibiotics, irrespective of treatment.

A comparator group of controls consisted of eligible participants whose LA resolved within 3 months after antibiotic initiation. Clinical resolution of LA was defined as having resolution of all pain and stiffness and no more than a small joint effusion on examination. We considered small, asymptomatic effusions as consistent with clinical resolution because (1) LA can cause prolonged (if mild) joint swelling even after apparent eradication of detectable borrelial infection, and (2) this clinical state was considered a suitable endpoint for both treatment and followup in all 3 centers.

Independent variables. Primary explanatory variables included demographics, clinical features at the time of diagnosis, and clinical features observed within 6 weeks of antibiotic initiation (Supplementary Table 1, available with the online version of this article). These variables were selected a priori based on the literature and investigators' clinical experiences. A 6-week window for certain variables was selected because (1) early clinical changes after treatment initiation could have important prognostic value, and (2) certain symptoms and signs suggesting chronicity might be recognized or reported only by specialists seeing patients who previously started treatment but did not respond. We also collected information on other demographic, clinical, and treatment characteristics evaluated in exploratory secondary analyses. We collected information about medication-associated toxicities while participants received antibiotics. Missing duration for the 4 main antibiotics (amoxicillin, doxycycline, ceftriaxone, and cefuroxime) was imputed at the median, 28 days (Supplementary Table 2, available with the online version of this article). This imputation was considered reasonable given the small proportion of courses lacking duration data and minimal variability in prescribing across sites, which broadly adhered to guidelines for treatment duration ${ }^{3,4}$. Variables with missing data (e.g., arthritis duration, antibiotic dose) were considered missing at random, given available data associated with missingness including center, specialty of diagnosing clinician, time to first rheumatology visit, and outcome. Thus, we imputed missing data by multiple imputation of 20 datasets using chained equations ${ }^{15}$

Data collection. Study data were abstracted from each center's EHR using standardized forms by an attending pediatric rheumatologist, pediatric rheumatology fellow with $\geq 1$ year of clinical training, or trained research staff. Charts extracted by nonclinical research staff were reviewed by supervising physicians to ensure accuracy. Data inconsistencies prompted repeat EHR review and data abstraction as needed. Clinical data related to care preceding the first visit to pediatric rheumatology clinic were extracted from primary documentation (e.g., primary care notes) where available or otherwise from rheumatologists' consultation notes. Data were collected and managed using REDCap (Research Electronic Data Capture) tools hosted at Nemours ${ }^{16}$.

Personal non-commercial use only. The Journal of Rheumatology Copyright $\odot$ (2019. All rights reserved 
Statistical analysis. Characteristics of cases and controls were compared using standard descriptive statistics. Primary explanatory variables associated with ARLA in univariate analyses $(p<0.2)$ were included in a multivariable logistic regression model. We retained model variables that were significantly associated with ARLA $(\mathrm{p}<0.05)$ or changed adjusted OR (aOR) between age and ARLA by $\geq 10 \%$. We did not adjust for multiple comparisons because each primary explanatory variable was hypothesis-driven. We tested for statistical interactions between age and other variables, considering $\mathrm{p}<0.1$ to represent a significant interaction. Exploratory secondary models incorporated additional covariates that were considered clinically relevant posthoc, such as characteristics of initial antibiotic treatment (e.g., dose, frequency).

We performed several sensitivity analyses to assess the influence of our assumptions: additional adjustment for treatment center; alternative outcome definition of persistent $L A \geq 6$ months after antibiotic initiation; exclusion of children without documented Lyme ELISA; exclusion of children with antibiotic courses of unknown duration; exclusion of children who did not receive IV antibiotic treatment despite lack of improvement on oral antibiotics, in accordance with treatment recommendations ${ }^{3}$; and categorization of participants who were lost to followup as (1) having ARLA (worst case imputation) or (2) having ARLA if (a) the imputed date of resolution was $\geq 2$ months after completion of sufficient treatment, or (b) there was insufficient treatment to qualify for ARLA before loss to followup.

All analyses were performed using Stata 12.1 (StataCorp LP). Two-sided $\mathrm{p}$ values $<0.05$ were considered significant.

\section{RESULTS}

Of 383 children with LA, 49 children developed ARLA (cases), and 188 children had arthritis that resolved within 3 months after starting antibiotics (controls; Figure 1). Each center contributed different numbers of cases $(n=28,7,14)$ corresponding to their respective sample size, but the percentage of subjects with ARLA did not significantly differ across centers $(18 \%, 23 \%, 30 \% ; \mathrm{p}=0.19)$. Of the remaining 146 children with LA, 107 had LA resolve $\geq 3$ months since starting antibiotics, and 39 were lost to followup before ARLA determination. Compared with children with faster resolution of arthritis, children with ARLA were more frequently older [median age $11.6 \mathrm{yrs}$ (interquartile range; IQR 9.0-13.8) vs 9.0 yrs (IQR 6.9-11.8)] and less likely to be seen by pediatric rheumatologists early in treatment (Table 1). Children with ARLA were more likely to present with prolonged continuous joint symptoms [median 15 days (IQR 5-45) vs median 5 days (IQR 2-10)] and arthritis affecting a single knee (90\% vs $63 \%$ ). In contrast, children whose arthritis resolved within 3 months were more likely to present with fever (24\% vs $16 \%$ ), elevated erythrocyte sedimentation rate (ESR; 35\% vs $16 \%$ ), and severe pain (32\% vs 6\%), sometimes leading to hospitalization ( $16 \%$ vs $6 \%$ ); $58 \%$ of controls had at least 1 feature of this severe phenotype versus $22 \%$ of cases. Many measured baseline characteristics did not differ between groups, including history of prior LD episodes or early LD symptoms, history of symptoms or diagnoses of autoimmune diseases, family history, or year of presentation. After starting antibiotics, children with ARLA were more likely to have markedly worsening arthritis, including new massive effusions, rupture of joint capsule or popliteal cyst

\section{4 charts reviewed}

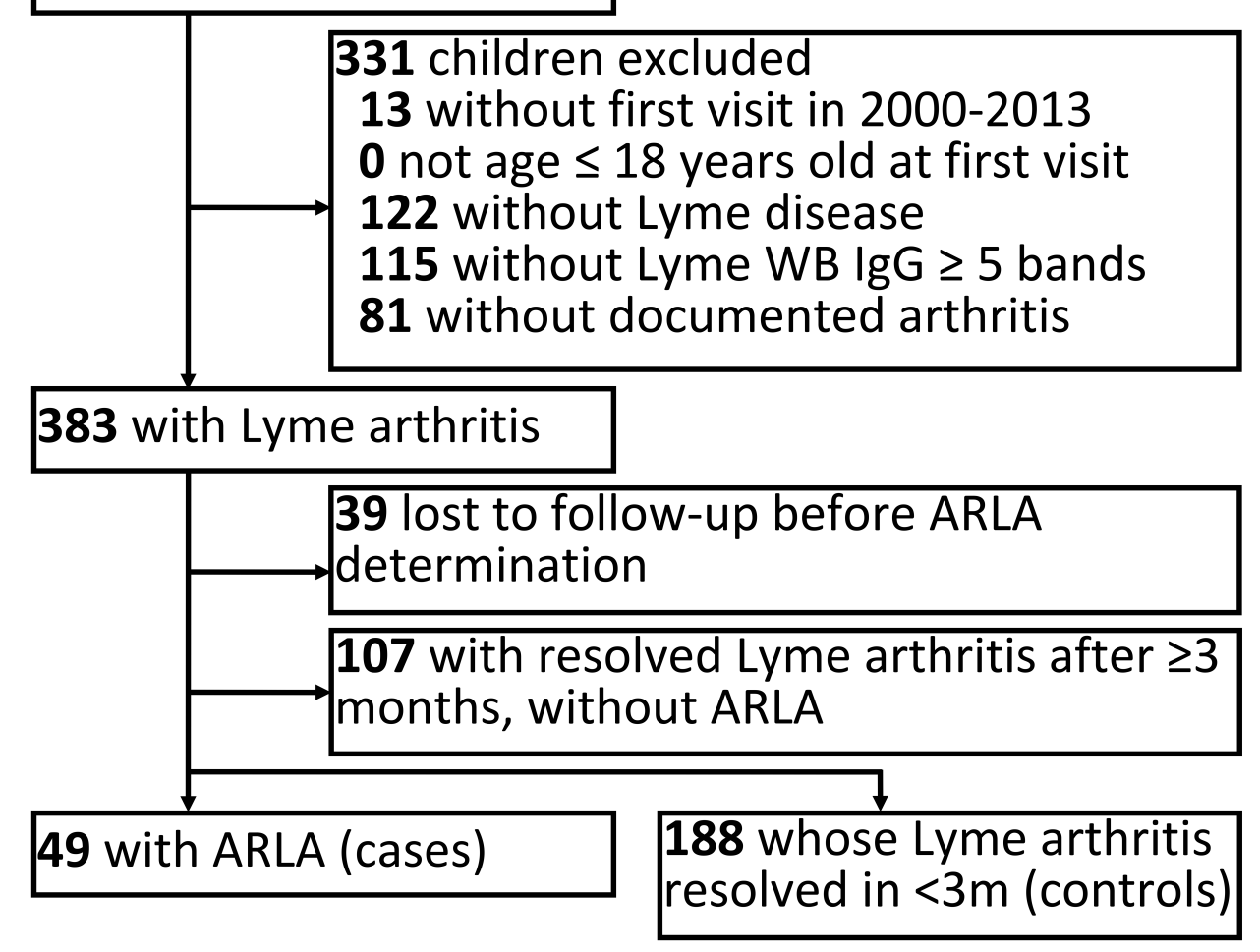

Figure 1. Subject selection diagram. This flowchart details the process of selecting 237 study participants (49 cases and 188 controls) after exclusion of 331 children without documented Lyme arthritis and 148 others who did not meet criteria for either cases or controls. ARLA: antibiotic-refractory Lyme arthritis; m: months; WB IgG: Western blot immunoglobulin G. 
Table 1. Characteristics of children with and without ARLA.

\begin{tabular}{|c|c|c|c|}
\hline Characteristics & Resolved $<3$ mos, $\mathrm{n}=188$ & ARLA, $\mathrm{n}=49$ & $\mathrm{p}^{1}$ \\
\hline \multicolumn{4}{|l|}{ Demographics } \\
\hline Age, yrs, median (IQR) & $9.0(6.9-11.8)$ & $11.6(9.0-13.8)$ & $<0.001^{2}$ \\
\hline Male sex ${ }^{3}$ & $121(64)$ & $29(59)$ & 0.50 \\
\hline Race & & & 0.05 \\
\hline White & $142(76)$ & $45(92)$ & \\
\hline Non-Hispanic & $158(84)$ & $47(96)$ & \\
\hline Hispanic & $8(4)$ & 0 & \\
\hline Unknown ethnicity & $22(12)$ & $2(4)$ & \\
\hline Year of presentation to rheumatology clinic & & & 0.51 \\
\hline $2000-2006$ & $56(30)$ & $17(35)$ & \\
\hline $2007-2013$ & $132(70)$ & $32(65)$ & \\
\hline Orthopedics & $32(17)$ & $12(24)$ & \\
\hline Infectious diseases & $1(1)$ & 0 & \\
\hline Seen by a pediatric rheumatologist within 1 week of antibiotic initiation & $67(36)$ & $3(6)$ & $<0.001$ \\
\hline Days after antibiotic initiation to presentation in rheumatology clinic, median (IQR) & $21(0-38)$ & $93(41-131)$ & $<0.001^{5}$ \\
\hline \multicolumn{4}{|l|}{ Clinical presentation } \\
\hline Acute migratory arthritis ${ }^{3}$ & $7(4)$ & 0 & $0.35^{4}$ \\
\hline Prior self-resolving episodes of joint swelling ${ }^{3}$ & $74(39)$ & $13(27)$ & 0.10 \\
\hline Continuous joint symptoms for at least 6 weeks ${ }^{3}$ & $4(2)$ & $8(16)$ & $<0.001^{4}$ \\
\hline Missing duration of baseline joint symptoms & $37(20)$ & $17(35)$ & 0.03 \\
\hline$\geq 2$ active joints ${ }^{3}$ & $51(27)$ & $2(4)$ & 0.001 \\
\hline Arthritis limited to knee $(s)^{3}$ & $138(73)$ & $46(94)$ & 0.002 \\
\hline Arthritis limited to a single knee & $118(63)$ & $44(90)$ & $<0001$ \\
\hline Severe phenotype $e^{3,6}$ & $109(58)$ & $11(22)$ & $<0.001$ \\
\hline History of previously treated Lyme disease & $12(6)$ & $3(6)$ & $0.99^{4}$ \\
\hline History of known tick bite & $34(18)$ & $8(16)$ & 0.82 \\
\hline History of personal autoimmune disease & $3(2)$ & 0 & $0.99^{4}$ \\
\hline Family history of Lyme disease & $18(10)$ & $9(18)$ & 0.08 \\
\hline Family history of other autoimmune disease & $76(40)$ & $24(49)$ & 0.28 \\
\hline \multicolumn{4}{|l|}{ Features within 6 weeks of diagnosis } \\
\hline Features of $\mathrm{SpA}^{3,7}$ & $2(1)$ & $1(2)$ & $0.50^{4}$ \\
\hline Clinical worsening on treatment ${ }^{3,8}$ & $9(5)$ & $10(20)$ & $0.001^{4}$ \\
\hline Presence of chronic joint changes ${ }^{3,9}$ & $39(21)$ & $6(12)$ & 0.18 \\
\hline Dose of first antibiotic course too low & $5(3)$ & $5(10)$ & $0.04^{4}$ \\
\hline Unknown dose of first antibiotic course & $16(9)$ & $7(14)$ & $0.28^{4}$ \\
\hline First course of antibiotic with too low frequency ${ }^{10}$ & $17(9)$ & $3(6)$ & $0.77^{4}$ \\
\hline Unknown frequency of first antibiotic course & $11(6)$ & $6(12)$ & $0.13^{4}$ \\
\hline Documented treatment nonadherence ${ }^{11}$ & $3(2)$ & $3(6)$ & $0.11^{4}$ \\
\hline Prescribed nonsteroidal antiinflammatories & $55(29)$ & $14(29)$ & 0.93 \\
\hline
\end{tabular}

Values are expressed as $\mathrm{n}(\%)$ unless otherwise indicated. ${ }^{1}$ Calculated from chi-square test except where indicated. ${ }^{2}$ Calculated from $\mathrm{t}$ test. ${ }^{3}$ Primary explanatory variable. ${ }^{4}$ Calculated from Fisher's exact test. ${ }^{5}$ Calculated from Wilcoxon rank-sum test. ${ }^{6}$ Characteristics combined into 1 factor of severity in multivariable models. ${ }^{7}$ Presence of inflammatory back pain, enthesitis, tendonitis, or dactylitis; personal history of psoriasis, inflammatory bowel disease, or acute anterior uveitis. ${ }^{8}$ Massive effusion, joint capsule rupture, or symptomatic joint recruitment after antibiotic initiation. ${ }^{9}$ Flexion contracture present or $>20^{\circ}$ in the presence of massive effusion, proximal muscle atrophy, condylar hypertrophy, or erosions on imaging. ${ }^{10}$ In all but 1 case, amoxicillin was given twice daily rather than 3 times daily, as recommended by treatment guidelines $s^{3,4} .{ }^{11}$ Defined as taking $<80 \%$ of prescribed antibiotic doses. ARLA: antibiotic-refractory Lyme arthritis; ESR: erythrocyte sedimentation rate; IAGC: intraarticular glucocorticoid; IgG: immunoglobulin G; IQR: interquartile range; SpA: spondyloarthritis. 
causing painful lower leg swelling, or symptomatic recruitment of additional arthritic joints (20\% vs 5\%).

Regarding treatment characteristics, children with ARLA were less likely to receive antibiotics at guideline-recommended doses (usually amoxicillin) and more likely to report not taking most or all prescribed antibiotic doses. In contrast, there were no reported differences in antibiotic frequency (amoxicillin thrice daily vs twice daily) or early use of daily nonsteroidal antiinflammatory drugs (NSAID; Table 1). Children with ARLA were more likely to have taken doxycycline, consistent with their older ages (Table 2). Notably, among children age $\geq 8$ years, a similar percentage of cases $(65 \%)$ and controls $(64 \%)$ took doxycycline as a first-line antibiotic $(\mathrm{p}=0.91)$. Over half of cases were prescribed $>10$ weeks of antibiotics, while controls were prescribed antibiotics for a median of 30 days (IQR 28-35). Cases were also more likely to receive IV antibiotics (57\% vs 7\%) and be prescribed NSAID at any point (80\% vs 30\%). Consistent with this higher treatment burden, reported adverse events during the antibiotic treatment period were more common among children with ARLA (37\% vs $15 \%)$, including rash ( $18 \%$ vs $4 \%$ ), headache ( $8 \%$ vs $1 \%$ ), and hospitalization for adverse events [6\% (3 cases) vs 1\% ( 1 control)]. Reasons for hospitalization of cases were allergic reaction with rash, abdominal pain and vomiting from suspected biliary sludging, and allergic reaction plus a mechanical IV line problem (all with ceftriaxone); 1 control taking amoxicillin was hospitalized for rash.

In primary multivariable analysis, 4 clinical factors were significantly associated with increased risk of ARLA: age $\geq 10$ years (aOR 2.5, 95\% CI 1.1-5.6), presence of continuous joint symptoms for $\geq 6$ weeks at diagnosis (aOR 9.4, 95\% CI 2.5-34.7), arthritis at diagnosis limited to one or both knees (aOR 5.1, 95\% CI 1.4-19.2), and clinical worsening on initial antibiotic treatment (aOR 4.2, 95\% CI 1.4-12.6; Table 3). In the same model, features of severe inflammation (fever, severe pain with or without hospitalization, or high ESR) were collectively associated with a decreased ARLA risk (aOR 0.4, 95\% CI 0.2-0.9). There was no evidence of statistical interaction between age and other model variables $(\mathrm{p}>0.1)$. In a separate model, 2 additional treatment-related factors - prescription of amoxicillin at subtherapeutic doses and poor reported antibiotic adherence - were also associated with ARLA (Table 3).

Table 2. Antibiotic use and treatment-associated adverse events among children with and without ARLA.

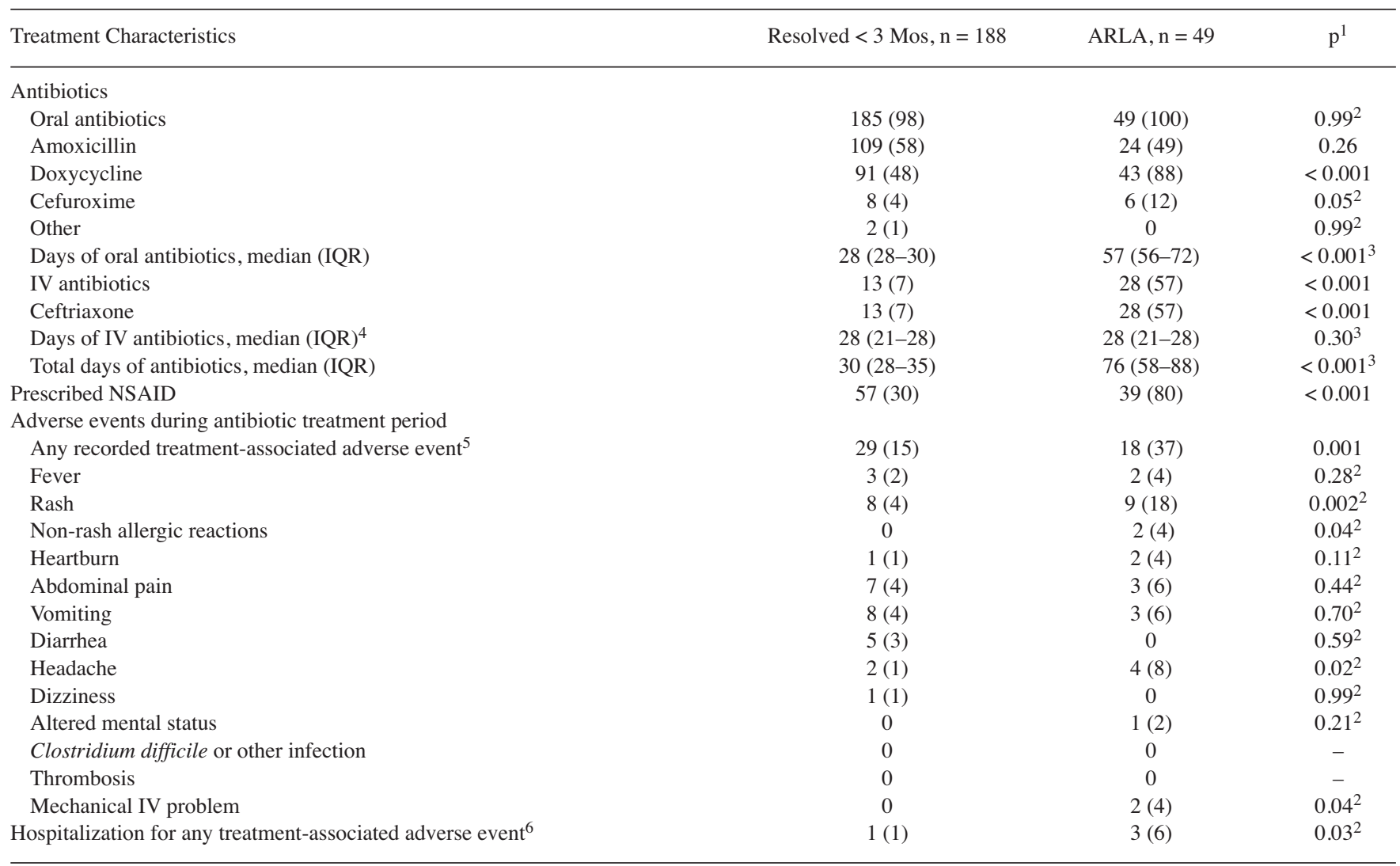

Values are expressed as $\mathrm{n}(\%)$ unless otherwise indicated. ${ }^{1}$ Calculated from chi-square test except where indicated. ${ }^{2}$ Calculated from Fisher's exact test. ${ }^{3}$ Calculated from Wilcoxon rank-sum test. ${ }^{4}$ Calculated among subjects who received ceftriaxone. ${ }^{5}$ Some subjects had $>1$ adverse event. ${ }^{6}$ Excluding hospitalization for worsening disease (e.g., leg swelling from popliteal cyst rupture). ARLA: antibiotic-refractory Lyme arthritis; IQR: interquartile range; IV: intravenous; NSAID: nonsteroidal antiinflammatory drugs. 
Univariate Analysis

Primary or Secondary

Explanatory Variable

Unadjusted OR (95\% CI)
Multivariable Analysis

Analysis of Primary Primary Explanatory

Explanatory Variables, Variables Plus Secondary $\mathrm{n}=237$ Adjusted $\mathrm{OR}^{1}(95 \% \mathrm{CI})$

\footnotetext{
Demographics

Age $\geq 10$ yrs

Male sex

$3.5(1.8-6.7)$

$0.8(0.4-1.6)$

Clinical features at presentation

Acute migratory arthritis

Prior, self-resolving episodes of joint swelling

Continuous joint symptoms for at least 6 weeks

Severe phenotype ${ }^{2}$

$\geq 9$ bands on Western blot

$\geq 2$ active joints

Arthritis limited to knee(s)

Premature IAGC injection

Clinical features within first 6 weeks after treatment initiation

Clinical worsening on treatment ${ }^{3}$

Features of $\mathrm{SpA}^{4}$

Presence of chronic joint changes 5

Exploratory treatment characteristics

Dose of first antibiotic course too low 6

Documented treatment non-adherence ${ }^{7}$

$1.8(0.9-3.7)$

$0.5(0.3-1.1)$

$13.9(4.0-48.6)$

$0.2(0.1-0.5)$

$1.2(0.6-2.5)$

$0.1(0.03-0.5)$

$5.6(1.7-18.9)$

$3.9(0.5-28.4)$

$5.0(1.9-13.1)$

$1.9(0.2-21.5)$

$0.5(0.2-1.3)$

$4.0(1.2-14.6)$

$4.0(0.8-20.2)$

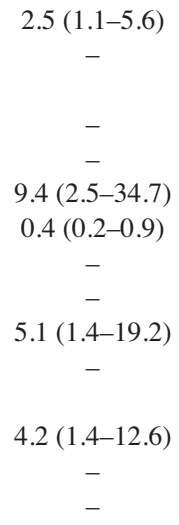

${ }^{1}$ Multivariable logistic models including all independent variables shown. ${ }^{2}$ Unexplained fever, severe pain, hospitalization for severe pain, or measured ESR $\geq 40 \mathrm{~mm} / \mathrm{h}$. ${ }^{3}$ Massive effusion, rupture of joint capsule or popliteal cyst, or symptomatic joint recruitment after antibiotic initiation. ${ }^{4}(1)$ Presence of inflammatory back pain, enthesitis (tenderness at bony insertions of tendons and ligaments), tendonitis, or dactylitis; or (2) personal history of psoriasis, inflammatory bowel disease, or acute anterior uveitis. ${ }^{5}$ (1) Flexion contracture $>20^{\circ}$ or presence of flexion contracture without massive effusion; (2) muscle atrophy proximal to involved joint; (3) hypertrophy of femoral condyles; (4) joint erosions on imaging. ${ }^{6}$ Antibiotic dose of first course too low per treatment guidelines ${ }^{3,4}$ ${ }^{7}$ Treatment non-adherence defined as reported consumption of < 80\% of prescribed antibiotic doses. ARLA: antibiotic-refractory Lyme arthritis; IAGC: intraarticular glucocorticoid; SpA: spondyloarthritis; ESR: erythrocyte sedimentation rate.
}

In sensitivity analyses, associations with all variables in the primary model remained consistent, but the effect size for certain variables was attenuated (Supplementary Table 3, available with the online version of this article).

Of 49 children with ARLA, 36 (73\%) received $\geq 2$ oral antibiotic courses. Fifteen of them subsequently received IV antibiotics. Nonantibiotic treatments (e.g., IAGC) and time to clinical resolution did not differ between children who did and did not receive ceftriaxone (Table 4). Six children had arthritis spread to new joints in the postantibiotic period, all of whom had received prior ceftriaxone $(p=0.03)$. Among children with ARLA, 4 of 5 children who received subtherapeutic oral antibiotics and 3 who were nonadherent to oral antibiotics also received ceftriaxone. Among 21 children who received oral but not IV antibiotics, 17 (81\%) experienced clinical improvement with oral antibiotics alone. Four children ( $8 \%$ of children with ARLA) did not receive ceftriaxone despite lack of improvement after 2 courses of oral antibiotics, including 1 child with subtherapeutic amoxicillin. Exclusion of these 4 cases did not substantively change our results (Supplementary Table 3, available with the online version of this article).

Among children with ARLA, half $(24,49 \%)$ had recurrent arthritis after full or clinical resolution of arthritis at a median of 273 (IQR 206-385) days after antibiotic initiation. Recurrences were more common after full, documented resolution of effusions (17) than in children with small, asymptomatic effusions (7). Children with fully resolved effusions did not differ from children with small, asymptomatic effusions in the number of prior antibiotic courses, number of prior IAGC injections, or time to first recurrence $(\mathrm{p}>0.1)$.

\section{DISCUSSION}

In a large multicenter cohort of pediatric LA, we identified 4 of 9 hypothesized factors that were associated with increased risk of antibiotic-refractory disease: older age, arthritis limited to the knee(s), prolonged continuous joint symptoms, and clinical worsening at initial treatment. Of several factors hypothesized to decrease ARLA risk, we found that children who favorably responded to antibiotics more often had fever, severe pain, or other signs of systemic inflammation. Notably, not only did children with ARLA receive antibiotics for longer periods of time, but over half were prescribed more than 10 weeks of antibiotics despite treatment guidelines recommending no more than $6-8$ weeks of antibiotics for

Personal non-commercial use only. The Journal of Rheumatology Copyright @ 2019. All rights reserved. 


\begin{tabular}{|c|c|c|c|c|}
\hline Characteristics & $\begin{array}{c}\text { ARLA, } \\
\mathrm{n}=49\end{array}$ & $\begin{array}{c}\text { ARLA, Did Not } \\
\text { Receive Ceftriaxone, } \\
\text { n }=21\end{array}$ & $\begin{array}{l}\text { ARLA, Received } \\
\text { Ceftriaxone, } \\
n=28\end{array}$ & $\mathrm{p}^{1}$ \\
\hline Any IAGC injection after antibiotic initiation ${ }^{3}$ & $32(65)$ & $14(67)$ & $18(64)$ & 0.86 \\
\hline 1 IAGC injection & $17(35)$ & $7(33)$ & $10(36)$ & \\
\hline 2 IAGC injections & $9(18)$ & $3(14)$ & $6(21)$ & \\
\hline Any IAGC injection after ceftriaxone or ARLA diagnosis (no ceftriaxone) & $28(57)$ & $14(67)$ & $14(50)$ & 0.24 \\
\hline Any prescribed NSAID after ceftriaxone or ARLA diagnosis (no ceftriaxone) & $34(69)$ & $17(81)$ & $17(61)$ & 0.13 \\
\hline Any DMARD 4 & $7(14)$ & $3(14)$ & $4(14)$ & 0.99 \\
\hline Synovectomy & $7(14)$ & $2(10)$ & $5(18)$ & $0.68^{5}$ \\
\hline Spread of arthritis to new joint after completion of antibiotics & $6(12)$ & 0 & $6(21)$ & $0.03^{5}$ \\
\hline
\end{tabular}

Values are expressed as $\mathrm{n}(\%)$ unless otherwise indicated. ${ }^{1} \mathrm{P}$ value calculated from chi-square test except where indicated. ${ }^{2} \mathrm{P}$ value calculated from Wilcoxon rank-sum test. ${ }^{3}$ IAGC not including premature injections before antibiotic initiation. ${ }^{4}$ Four children received methotrexate, 4 children received sulfasalazine, and 1 child received hydroxychloroquine; some children received $>1$ DMARD, and no child received a tumor necrosis factor inhibitor. ${ }^{5} \mathrm{P}$ value calculated from Fisher's exact test. ARLA: antibiotic-refractory Lyme arthritis; IQR: interquartile range; IAGC: intraarticular glucocorticoid; NSAID: nonsteroidal antiinflammatory drug; DMARD: disease-modifying antirheumatic drug.

$\mathrm{LA}^{3}$. The more intensive, prolonged treatment of children with ARLA was associated with higher rates of treatment-associated adverse events, including hospitalizations.

Our findings support prior research suggesting older age as a risk factor for ARLA in children $6,7,8$. The biologic basis for this finding is unclear. Doxycycline is used almost exclusively in older children and adolescents because of perceived risk of dental staining at earlier ages, but initial antibiotic choice did not explain the increased risk with age. Multiple lines of evidence suggest ARLA may be a postinfectious autoimmune condition, including associations of ARLA with HLA-DR $4{ }^{17,18}$, genetic polymorphisms relating to immune response $^{19}$, autoantibodies ${ }^{20}$, and pathogenic changes in regulatory $\mathrm{T}$ cells ${ }^{21,22}$ and Th17 cells ${ }^{23}$. ARLA may also result from immune dysregulation ${ }^{24}$ or abnormal immune response to persistent articular borrelial antigens ${ }^{25,26}$. The greater risk of older children to develop ARLA could relate to factors underlying the rising incidence of other autoimmune diseases in adolescence ${ }^{27}$. Further investigation should clarify whether the greater risk of ARLA among older children relates to one or more of these potential mechanisms. Our study also echoes previous research suggesting that new joint recruitment while taking antibiotics is a risk factor for ARLA in children ${ }^{8}$. Unlike the previous study, our definition of clinical worsening while receiving treatment also encompassed the development of massive effusions and rupture of large popliteal cysts, which we have observed clinically as other early prognosticators of treatment resistance.

Previous studies have not found any relationship between the duration of joint symptoms at diagnosis and ARLA in children $^{6,7,10}$ or in a predominantly adult cohort ${ }^{5}$. We hypothesized that sustained arthritis at diagnosis would be associated with ARLA based on clinical experience. The positive finding in our study could relate to its larger sample size or differences in the study population (e.g., pediatric vs adult, United States vs Europe ${ }^{28}$ ) or design (comparators responding within 3 months) and bears replication. Others have observed that younger children with LA more likely present with fever and pain ${ }^{6}$, but our results suggest that systemic inflammatory response is a marker of favorable response to antibiotics irrespective of age. The mechanism behind this finding is unclear but may relate to more effective spirochetal killing among patients with high early levels of Th1- or Th17-associated cytokines ${ }^{29}$. Further, the association between knee-only arthritis and ARLA is also a novel, previously unreported finding.

We were unable to substantiate the previous observation that premature IAGC increases ARLA risk ${ }^{5,11}$. The few children we identified with early injections limited our ability to study this potential risk factor. We did find that children treated with insufficient doses of amoxicillin were more likely to develop ARLA, lending support for the recommended daily dosage in pediatric $\mathrm{LA}^{3,4}$. In contrast, outcomes did not differ when amoxicillin was prescribed twice daily instead of 3 times daily, as per treatment recommendations ${ }^{3,4}$. Notably, there was little deviation from guidelines in the prescribed dosage or frequency of doxycycline or ceftriaxone.

Treatment guidelines recommend that patients whose LA does not respond to oral antibiotics receive IV antibiotics ${ }^{3}$. Most patients in our cohort (all but 4) received care consistent with these recommendations, and exclusion of these 4 individuals from regression models did not change our findings. Further, children who received only oral antibiotics had similar outcomes compared with children who received IV ceftriaxone, except that children who received IV antibiotics were more likely to experience new postantibiotic joint recruitment. Our results support the notion that IV antibiotics may be required for adequate spirochetal killing

$$
\text { Personal non-commercial use only. The Journal of Rheumatology Copyright (C) 2019. All rights reserved. }
$$


in children who do not respond to oral antibiotics, even though IV antibiotic therapy does not prevent ARLA or the need for nonantibiotic treatment for all children. Given the lack of high-quality evidence about second-line treatment regimens, the risks of IV antibiotics, and the potential benefit of early IAGC injection for $\mathrm{LA}^{30}$, pediatricians should consider referring children to pediatric rheumatologists for LA that remains persistently active after 2 antibiotic courses.

Our study has several strengths. We assembled the largest cohort of pediatric LA to date across several referral centers in a Lyme-endemic region, giving us greater statistical power and generalizability to identify important clinical factors. Unlike similar previous studies, we focused on factors hypothesized to relate to ARLA, making our findings less likely to result from chance alone, and thus more scientifically credible. In addition, our study highlights the potential dangers of overtreating LA with antibiotics after the infection itself is cleared and antibiotics are ineffective $e^{5,31,32}$. This treatment-related risk is noteworthy because, in other settings, antibiotic use and antibiotic-associated dysbiosis may contribute to the development of acute and chronic arthritis in children ${ }^{33,34}$.

Our study also has several limitations. Given the setting at pediatric rheumatology referral centers, the study population and the high proportion of children with ARLA were not representative of all pediatric LA. For this reason, certain factors that we identified may relate to patterns of rheumatology referral more than ARLA risk, and our results may not generalize to all children with LA. Nonetheless, children with persistently active LA are commonly referred to pediatric rheumatologists, and results were similar across 3 centers with geographically and demographically distinct referral populations. A population-based case-control or cohort study would help further validate our findings. Another limitation was the high prevalence of missing data for certain variables, including baseline symptom duration, a primary explanatory variable associated with ARLA. Many children with ARLA were first seen in participating rheumatology clinics months after diagnosis, which led to higher rates of missing early clinical data. Nonetheless, we used available clinical data and referral patterns to impute missing data, and our findings were robust across multiple sensitivity analyses. Incomplete early documentation among many cases may also have underestimated nonadherence and the actual burden of early treatment toxicity. Finally, not all children were followed until full resolution of joint effusions, compatible with the clinical practice of participating centers. We found no evidence that recurrent arthritis was more common after incomplete resolution of effusions.

We identified several factors associated with the development of ARLA in children referred to pediatric rheumatologists, including older age, prolonged joint symptoms at diagnosis, knee-only arthritis, and clinical worsening on initial treatment. Children presenting with fevers or severe pain generally have more rapid and favorable response to antibiotics. Children with ARLA frequently receive multiple antibiotic courses, often exceeding current guidelines, resulting in more treatment-related toxicity. For children with persistently active LA after 2 antibiotic courses, pediatricians should consider starting antiinflammatory treatment and referring to a pediatric rheumatologist.

\section{ACKNOWLEDGMENT}

The authors thank Jenna Tress for assisting with regulatory documentation and data collection. The authors also thank Meredith Buckley, Kelly Collier, Janille Diaz, Elizabeth Kaufman, Valerie Levy, Bernadette Lewcun, and Amanda Schlefman for collecting data.

\section{ONLINE SUPPLEMENT}

Supplementary material accompanies the online version of this article.

\section{REFERENCES}

1. Schwartz AM, Hinckley AF, Mead PS, Hook SA, Kugeler KJ. Surveillance for Lyme disease - United States, 2008-2015. MMWR Surveill Summ 2017;66:1-12.

2. Gerber MA, Shapiro ED, Burke GS, Parcells VJ, Bell GL. Lyme disease in children in southeastern Connecticut. Pediatric Lyme Disease Study Group. N Engl J Med 1996;335:1270-4

3. Wormser GP, Dattwyler RJ, Shapiro ED, Halperin JJ, Steere AC, Klempner MS, et al. The clinical assessment, treatment, and prevention of Lyme disease, human granulocytic anaplasmosis, and babesiosis: clinical practice guidelines by the Infectious Diseases Society of America. Clin Infect Dis 2006;43:1089-134.

4. American Academy of Pediatrics. Lyme disease. In: Kimberlin DW, Brady MT, Jackson MA, Long SS, editors. Red book: 2015 report of the Committee on Infectious Diseases. 30th ed. Elk Grove Village: American Academy of Pediatrics; 2015:516-25.

5. Steere AC, Angelis SM. Therapy for Lyme arthritis: strategies for the treatment of antibiotic-refractory arthritis. Arthritis Rheum 2006;54:3079-86.

6. Bentas W, Karch H, Huppertz HI. Lyme arthritis in children and adolescents: outcome 12 months after initiation of antibiotic therapy. J Rheumatol 2000;27:2025-30.

7. Tory HO, Zurakowski D, Sundel RP. Outcomes of children treated for Lyme arthritis: results of a large pediatric cohort. J Rheumatol 2010;37:1049-55.

8. Groh B, Ahn N. Lyme arthritis outcomes in children: a single center cohort study [abstract]. Pediatr Rheumatol 2012;Suppl 1:A38.

9. Brescia AC, Rose CD, Fawcett PT. Prolonged synovitis in pediatric Lyme arthritis cannot be predicted by clinical or laboratory parameters. Clin Rheumatol 2009;28:591-3.

10. Nimmrich S, Becker I, Horneff G. Intraarticular corticosteroids in refractory childhood Lyme arthritis. Rheumatol Int 2014;34:987-94.

11. Huppertz HI, Karch H, Suschke HJ, Döring E, Ganser G, Thon A, et al. Lyme arthritis in European children and adolescents. The Pediatric Rheumatology Collaborative Group. Arthritis Rheum 1995;38:361-8

12. Klempner MS, Hu LT, Evans J, Schmid CH, Johnson GM, Trevino $\mathrm{RP}$, et al. Two controlled trials of antibiotic treatment in patients with persistent symptoms and a history of Lyme disease. $\mathrm{N}$ Engl $\mathrm{J}$ Med 2001;345:85-92.

13. Berende A, ter Hofstede HJ, Vos FJ, van Middendorp H, Vogelaar ML, Tromp M, et al. Randomized trial of longer-term therapy for symptoms attributed to Lyme disease. N Engl J Med 2016;374:1209-20

14. Oksi J, Nikoskelainen J, Hiekkanen H, Lauhio A, Peltomaa M, Pitkäranta A, et al. Duration of antibiotic treatment in disseminated

Personal non-commercial use only. The Journal of Rheumatology Copyright $\odot$ 2019. All rights reserved. 
Lyme borreliosis: a double-blind, randomized, placebo-controlled, multicenter clinical study. Eur J Clin Microbiol Infect Dis 2007;26:571-81.

15. Sterne JA, White IR, Carlin JB, Spratt M, Royston P, Kenward MG, et al. Multiple imputation for missing data in epidemiological and clinical research: potential and pitfalls. BMJ 2009;338:b2393.

16. Harris PA, Taylor R, Thielke R, Payne J, Gonzalez N, Conde JG. Research electronic data capture (REDCap) - a metadata-driven methodology and workflow process for providing translational research informatics support. J Biomed Inform 2009;42:377-81.

17. Steere AC, Dwyer E, Winchester R. Association of chronic Lyme arthritis with HLA-DR4 and HLA-DR2 alleles. N Engl J Med 1990;323:219-23.

18. Kalish RA, Leong JM, Steere AC. Association of treatment-resistant chronic Lyme arthritis with HLA-DR4 and antibody reactivity to OspA and OspB of Borrelia burgdorferi. Infect Immun 1993;61:2774-9.

19. Strle K, Shin JJ, Glickstein LJ, Steere AC. Association of a Toll-like receptor 1 polymorphism with heightened Th1 inflammatory responses and antibiotic-refractory Lyme arthritis. Arthritis Rheum 2012;64:1497-507.

20. Londoño D, Cadavid D, Drouin EE, Strle K, McHugh G, Aversa JM, et al. Antibodies to endothelial cell growth factor and obliterative microvascular lesions in the synovium of patients with antibiotic-refractory Lyme arthritis. Arthritis Rheumatol 2014;66:2124-33.

21. Shen S, Shin JJ, Strle K, McHugh G, Li X, Glickstein LJ, et al. Treg cell numbers and function in patients with antibiotic-refractory or antibiotic-responsive Lyme arthritis. Arthritis Rheum 2010; 62:2127-37.

22. Vudattu NK, Strle K, Steere AC, Drouin EE. Dysregulation of CD4+CD25(high) $\mathrm{T}$ cells in the synovial fluid of patients with antibiotic-refractory Lyme arthritis. Arthritis Rheum 2013; 65:1643-53.

23. Strle K, Sulka KB, Pianta A, Crowley JT, Arvikar SL, Anselmo A, et al. T-helper 17 cell cytokine responses in Lyme disease correlate with Borrelia burgdorferi antibodies during early infection and with autoantibodies late in the illness in patients with antibiotic-refractory Lyme arthritis. Clin Infect Dis 2017;64:930-8.
24. Lochhead RB, Strle K, Kim ND, Kohler MJ, Arvikar SL, Aversa $\mathrm{JM}$, et al. MicroRNA expression shows inflammatory dysregulation and tumor-like proliferative responses in joints of patients with postinfectious Lyme arthritis. Arthritis Rheumatol 2017;69:1100-10.

25. Bockenstedt LK, Gonzalez DG, Haberman AM, Belperron AA. Spirochete antigens persist near cartilage after murine Lyme borreliosis therapy. J Clin Invest 2012;122:2652-60.

26. Wormser GP, Nadelman RB, Schwartz I. The amber theory of Lyme arthritis: initial description and clinical implications. Clin Rheumatol 2012;31:989-94.

27. Nigrovic PA, Raychaudhuri S, Thompson SD. Review: genetics and the classification of arthritis in adults and children. Arthritis Rheumatol 2018;70:7-17.

28. Li X, Strle K, Wang P, Acosta DI, McHugh GA, Sikand N, et al. Tick-specific borrelial antigens appear to be upregulated in American but not European patients with Lyme arthritis, a late manifestation of Lyme borreliosis. J Infect Dis 2013;208:934-41.

29. Strle K, Stupica D, Drouin EE, Steere AC, Strle F. Elevated levels of IL-23 in a subset of patients with post-Lyme disease symptoms following erythema migrans. Clin Infect Dis 2014;58:372-80.

30. Horton DB, Taxter AJ, Davidow AL, Groh B, Sherry DD, Rose CD Intraarticular glucocorticoid injection as second-line treatment for Lyme arthritis in children. J Rheumatol 2019 (accepted for publication).

31. Nocton JJ, Dressler F, Rutledge BJ, Rys PN, Persing DH, Steere AC. Detection of Borrelia burgdorferi DNA by polymerase chain reaction in synovial fluid from patients with Lyme arthritis. N Engl J Med 1994;330:229-34.

32. Carlson D, Hernandez J, Bloom BJ, Coburn J, Aversa JM, Steere AC. Lack of Borrelia burgdorferi DNA in synovial samples from patients with antibiotic treatment-resistant Lyme arthritis. Arthritis Rheum 1999;42:2705-9.

33. Horton DB, Scott FI, Haynes K, Putt ME, Rose CD, Lewis JD, et al. Antibiotic exposure and juvenile idiopathic arthritis: a case-control study. Pediatrics 2015;136:e333-43.

34. Horton DB, Strom BL, Putt ME, Rose CD, Sherry DD, Sammons JS. Epidemiology of Clostridium difficile infection-associated reactive arthritis in children: an underdiagnosed, potentially morbid condition. JAMA Pediatr 2016;170:e160217. 